\title{
Already, But Not Yet: Ending Unethical Practices in Authorship
}

\author{
Young-Chul Jung \\ Department of Psychiatry, Yonsei University College of Medicine, Seoul, Republic of Korea
}

\begin{abstract}
There was a national anger outburst after the South Korean government reported that at least 82 published scientific papers written by researchers included their children-most of whom were in middle school or high school-as coauthors. ${ }^{1}$ The practice, in most of cases, was thought to be intended to give their children an advantage and improve their chances to be accepted in university admissions. Although this kind of child authorship scandal is unprecedented, we are already used to unethical practices and problems related to authorship in academic research.
\end{abstract}

One of the most common ethical issues with authorship is "honorary authorship," which refers to inclusion of individuals as authors even when the actual contribution to the manuscript is insufficient. According to a new study ${ }^{2}$ analyzing survey responses from more than 12,000 American scholars in 18 disciplines, $35.5 \%$ of respondents admitted giving at least one honorary authorship to a researcher who contributed little to the paper. According to this study, the probability of honorary authorship increased by $90 \%$ and $40 \%$ in assistant professors and associate professors compared to full professors. Medicine, together with management and ecology were disciplines where honorary authors were statistically more likely to be included; whereas political science, mathematics, chemistry and economics were statistically lower than average. The most common reason of honorary authorship was because the individual was the director of the lab or in a position of authority that could affect the scholar's career. As most of these results are disturbing, what bothers us most is that we have to admit that these responses would be similar, if not worse, among scholars in South Korea.

Right after the 2005 Hwang Woo-Suk scandal, which attracted attention of the whole country for fabricating experiments and including improper coauthors, the number of authors per paper in medical science dropped to an average of 3.7 authors for a short period from 2005 to 2007 in South Korea; however, this didn't last long. In the era of large-scale collaborative research projects, the number of authors are steadily

(c) This is an Open Access article distributed under the terms of the Creative Commons Attribution Non-Commercial License (http://creativecommons.org/licenses/by$\mathrm{nc} / 4.0$ ) which permits unrestricted non-commercial use, distribution, and reproduction in any medium, provided the original work is properly cited. increasing; and defining authorship have been even more complex. Regarding South Korean projects with international collaborations, the average number of authors in the top $10 \%$ medical science journals were 14.7 in $2015 .^{3}$ The International Committee of Medical Journal Editors ${ }^{4}$ recommends each author to meet all of the four criteria: 1) Substantial contributions to the conception or design of the work: or the acquisition, analysis, or interpretation of data for the work; AND 2) Drafting the work or revising it critically for important intellectual content; AND 3) Final approval of the version to be published; AND 4) Agreement to be accountable for all aspects of the work in ensuring that questions related to the accuracy or integrity of any part of the work appropriately investigated and resolved. However, as the scale of multicenter multidisciplinary collaboration keeps expanding, sometimes it feels unrealistic to expect each coauthor to actually participate in writing the manuscript.

Even though many journals have updated their policies to discourage unethical practices in authorship, such as the contributor model ${ }^{5}$ the effectiveness of most policies largely depends on the willingness of scholars to be honest about their respective contributions. ${ }^{6}$ It is not hard to figure the minds of the parents who listed their children as authors, as well as the minds of the students who included their mentors as authors. But that's why we all have to keep our own minds up to root out these unethical practices.

\section{REFERENCES}

1. Zastrow M. Kid co-authors in South Korea spur government probe. Nature 2018;554:154-155.

2. Fong EA, Wilhite AW. Authorship and citation manipulation in academic research. PLoS One 2017;12:e0187394.

3. Jang H, Kim, K, Huh S, Kim H. Increasing number of authors per paper in Korean science and technology papers. Sci Ed 2016;3:80-89.

4. Defining the Role of Authors and Contributors. Available at: http:// www.icmje.org/recommendations/browse/roles-and-responsibilities/ defining-the-role-of-authors-and-contributors.html. Accessed March 16, 2018.

5. Resnik DB. A proposal for a new system of credit allocation in science. Sci Eng Ethics 1997;3:237-243.

6. Borenstein J, Shamoo AE. Rethinking authorship in era of collaborative research. Account Res 2015;22:267-283. 